\title{
Circular dichroism and Raman optical activity in antiferromagnetic transition metal fluorides
}

\author{
K.R. Hoffman \\ Department of Physics, Whitman College, Walla Walla, WA 99362, USA \\ D.J. Lockwood \\ Institute for Microstructural Sciences, National Research Council, Ottawa, ON \\ K1A 0R6, Canada \\ E-mail: david.lockwood@nrc.ca \\ W.M. Yen \\ Department of Physics and Astronomy, University of Georgia, Athens, GA 30602, USA
}

Received February 17, 2005

\begin{abstract}
The Raman optical activity (ROA) of magnons in rutile-structure antiferromagnetic $\mathrm{FeF}_{2}$ $\left(T_{N}=78 \mathrm{~K}\right)$ has been studied as a function of temperature and applied magnetic field. For exciting light incident along the $c$ axis, ROA is observed for magnons but not for phonons. In zero field, a small splitting $\left(0.09 \mathrm{~cm}^{-1}\right)$ of the two acoustic-magnon branches is observed for the first time by inelastic light scattering. The splitting in applied magnetic field is found to reduce with increasing temperature in accordance with theory. No ROA was detected for two-magnon excitations. In optical absorption measurements performed over thirty years ago, a very small circular dichroism (CD) was observed in the magnon sidebands of other simple rutile antiferromagnetic fluorides $\left(\mathrm{MnF}_{2}\right.$ and $\left.\mathrm{CoF}_{2}\right)$. The origin of this $\mathrm{CD}$ was not understood at the time. The Raman studies of the one-magnon Raman scattering in $\mathrm{FeF}_{2}$ have demonstrated that in zero field the degeneracy of the antiferromagnetic magnon branches is lifted by a weak magnetic dipole-dipole interaction, as predicted by Pincus and Loudon and by White four decades ago. The source of the observed CD in the magnon sidebands can now be traced to this same magnetic-dipole induced splitting.
\end{abstract}

PACS: 75.30.Ds, 75.30.Gw, 75.50.Ee, 78.20.Ls, 78.30.Hv

\section{Introduction}

In 1965, the discovery of the one-magnon sideband in the visible absorption spectrum of $\mathrm{MnF}_{2}$ [1] and the two-magnon absorption of the far-infrared spectrum of $\mathrm{FeF}_{2}$ [2] spurred many investigations into the optical properties of antiferromagnetic insulators. The theoretical framework regarding these two processes was developed by Tanabe et al. [3] and Allen et al. [4] In these materials, coupling between the various intrinsic excitations of the lattice, i.e., excitons, magnons and phonons, leads to the formation of cooperative transitions which can account for much of the spectral structure. An article discussing this work appears elsewhere in this special issue of Low Temperature Physics. In addition to absorption and lumines- cence measurements, magnon lines were also observed in Raman scattering measurements [5,6]. Raman scattering measurements permit the physical properties of magnons to be studied without the additional contributions due to electronic states associated with optical emission or absorption. A comprehensive review of Raman theory and experimental results for antiferromagnets is presented in the book by Cottam and Lockwood [7].

The magnetic character of the magnon transitions led to the utilization of circularly polarized light to explore the dichroic properties of these materials. In the early 1970's, a series of papers reported on their magnetic circular dichroism (MCD) in an applied magnetic field [8-10]. The authors reported at that time the observation of circular dichroism (CD) in 
certain magnon sidebands of $\mathrm{MnF}_{2}$ [8] and $\mathrm{CoF}_{2}$ [10] but not in $\mathrm{FeF}_{2}$ [9]; $\mathrm{CD}$ occurs in the absence of an applied magnetic field and corresponds to zero field MCD though the origins of the dichroism may arise from different sources. The authors were able to eliminate experimental artifacts as the source of the observed $\mathrm{CD}$, but were not able at that time to identify the physical basis for the unexpected observation. Recently, we revisited this problem and showed that the source of the CD is to be found in the dipolar interactions that exist between the antiferromagnetic sublattices, which render them inequivalent [11].

Raman optical activity (ROA) characterizes the difference in the Raman line intensity when excited with right and left circularly polarized light. The earliest experiments to combine Raman spectroscopy with chirality in solids were performed in the 1970's on CdS under resonance excitation conditions [12]. Attempts were also made to observe a similar effect in antiferromagnetic fluoride materials but the experimental sensitivity was insufficient. At about the same time ROA was also measured in a chiral molecule [13]. The chiral structure of some molecules makes them naturally suited to couple more strongly to either right or left circularly polarized light. The theoretical [14] and experimental developments in this field have been reviewed in the literature $[15,16]$. A series of Raman papers in the 1980's on the magnetic properties of dilute magnetic semiconductors employed the use of circular polarization to isolate spin flip transitions of Mn ions $[17,18]$. The improvement in detector sensitivity over a period of twenty years afforded us the opportunity in the 1990's to revisit the question of ROA in antiferromagnetic insulators. We were able to observe $\mathrm{ROA}$ in the one-magnon line of $\mathrm{FeF}_{2}$ [19-21] and several phonon lines in rutile structure materials $[22,23]$. In addition, we demonstrated for the first time that Raman scattering could be used to measure a zero field splitting of the magnon branches in antiferromagnetic $\mathrm{FeF}_{2}$ [24]. These results were then utilized to reinterpret the $\mathrm{CD}$ measurements completed in the early 1970's that were alluded to above [11].

In the following Sections, we present our current understanding of the dichroic properties of antiferromagnetic insulators possessing the rutile structure. The focus of this review will be on ROA measurements to explore the magnetic properties of these materials. We start with a summary of the zero field dichroism measurements in $\mathrm{MnF}_{2}, \mathrm{CoF}_{2}$, and $\mathrm{FeF}_{2}$ because the $\mathrm{CD}$ studies prompted one of us (WMY) to explore the use of ROA in antiferromagnetic fluorides. We then present a more thorough review of the ROA measurements, techniques, and analysis in $\mathrm{FeF}_{2}$. Here we mention first the work on phonons before focusing on the one- and two-magnon scattering results, including a revised assessment of the results from the temperature dependence and zero magnetic field studies of $\mathrm{FeF}_{2}$.

\section{Optical dichroism in rutile antiferromagnets}

Dichroism in a material is defined as the difference in the absorption coefficient of a medium for two related polarizations; thus $\mathrm{CD}$ arises from the difference in the absorption for right- and left-circularly polarized radiation. When these differences are induced by the application of an external magnetic field, the effect is termed MCD. In both CD and MCD, a unique direction is necessary in order to define the sense of circulation in the circularly polarized light; this is provided by some unique crystalline axis or by the direction of the applied magnetic field. Materials showing CD are normally called «optically active» and such crystals are relatively rare, whereas MCD is quite common in paramagnetic systems possessing a unique axis. MCD is conjugated to Faraday rotation by the Kramers-Kronig relations and constitutes the resonant rather than the dispersive phenomena in the transformation.

The compounds that we wish to discuss here are the common difluorides, $\mathrm{MnF}_{2}, \mathrm{CoF}_{2}$, and $\mathrm{FeF}_{2}$, all of which possess the rutile crystal structure. Below the Neel temperature, $T_{N}$, these materials are describable as simple two sublattice antiferromagnets and in principle they are ideal systems on which to study magnetically dichroic phenomena. The ground states of ions in each sublattice as well as the collective magnetic excitations (magnons) are identical to each other in every respect except their polarization. The sole remaining energy degeneracy, which will respond to an applied magnetic field, is that of the two sublattices; hence, the origin of magnetic dichroism in these systems is directly related to the lifting of this energy equivalence both in the electronic (exciton), the spin (magnon) or the combined (magnon sideband) systems.

For simple antiferromagnets in their ordered state, the ground state of each sublattice is non-degenerate and is identical to the other except for their sense of magnetization with respect to the unique crystalline axis. If the state for the «up» or $A$ sublattice is given by $|L, M\rangle$ then the corresponding «down» or $B$ sublattice is represented by $|L,-M\rangle$. For pure electronic transitions, the appropriate dipolar transition operators for circular polarized radiation transform as $L_{ \pm}+2 S_{ \pm}$where the $(+)$and $(-)$subscripts refer to right and left circular polarizations, respectively. If ions in one of the sublattices have a non-vanishing transition probability between two states for one of these operators, then the ions in the opposite sublattice will have an identical element for the corre- 
sponding conjugated operator. In the absence of a magnetic field, the sublattices are degenerate in energy and no CD should be observed. However, weak interactions between the sublattices, such as magnetic dipole-dipole interactions, can produce a small anisotropy between the two sublattices that results in CD. At low temperatures, a very small zero-field $\mathrm{CD}$ signal was observed in two of the three compounds investigated and it is the origin of these signals that will be addressed in this paper.

\section{Experimental results for circular dichroism measurements}

A finite $\mathrm{CD}$ was observed at low temperatures in two of the three difluorides studied. Signals were observed in the magnon sidebands of the ${ }^{6} A_{1 g}$ ground state to ${ }^{4} T_{1 q}$ and $\left({ }^{4} A_{1 g},{ }^{4} E_{q}\right)$ absorptions of $\mathrm{MnF}_{2}$ and of the ${ }^{4} T_{1}$ to ${ }^{2} A,{ }^{2} T_{1}\left({ }^{2} P\right)$ of $\mathrm{CoF}_{2}$; no $\mathrm{CD}$ signals were observed in $\mathrm{FeF}_{2}$. As noted earlier, the origins of $\mathrm{CD}$ and MCD are different; as a consequence, $\mathrm{CD}$ can be subtracted from MCD in order to determine the magnetic field induced splittings. When this is done the, the residual or pure MCD signal can be fitted accurately with a differential line shape; this indicates that in our cases the MCD arise simply from the lifting of the sublattice degeneracy, as per our expectation.

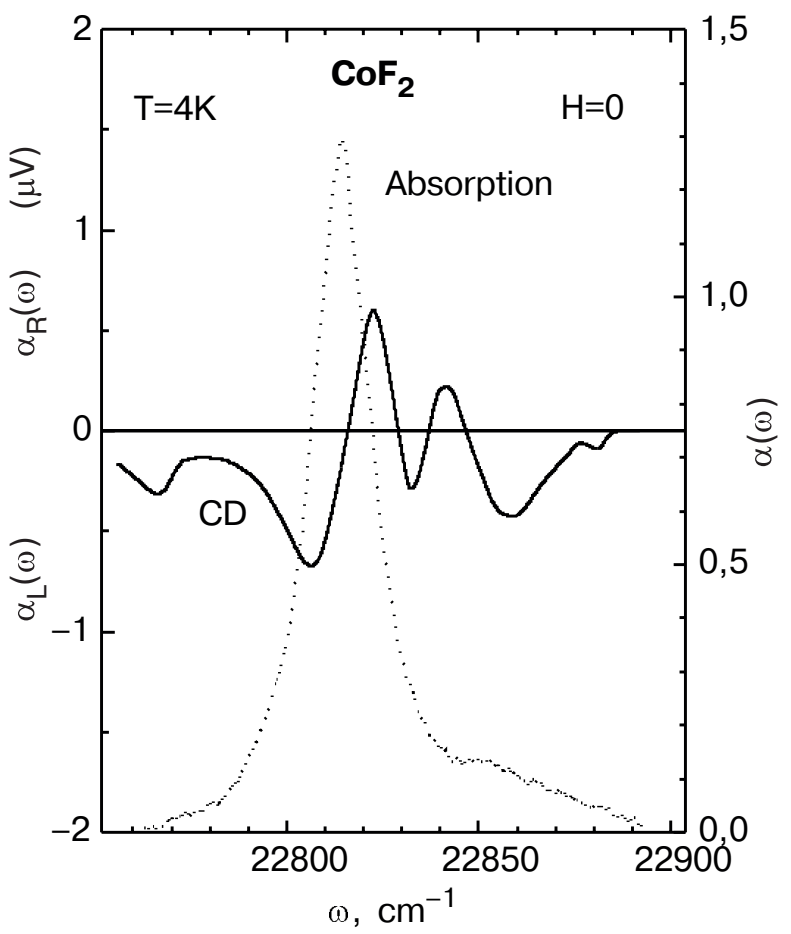

Fig. 1. The CD spectrum of the one-magnon sideband near $22,800 \mathrm{~cm}^{-1}$ of $\mathrm{CoF}_{2}$ at $4 \mathrm{~K}$ and the corresponding absorption spectrum of the sideband.
Figure 1 shows the absorption of a magnon sideband of the $\left({ }^{2} A,{ }^{2} T_{1}\right)$ transition of $\mathrm{CoF}_{2}$ along with the observed $\mathrm{CD}$ and MCD in a $2 \mathrm{~T}$ applied magnetic field. The CD is also purely differential in shape and is estimated to be an additive intrinsic splitting of the order of $\Delta \omega=0.015 \mathrm{~cm}^{-1}$. The $\mathrm{CD}$ in $\mathrm{CoF}_{2}$ becomes unobservable in the vicinity of $10 \mathrm{~K}$.

Figure 2 shows the absorption spectrum of the so-called $\sigma_{1}$ and $\sigma_{2}$ sidebands accompanying the ground state to ${ }^{4} T_{1 \mathrm{~g}}$ transition in $\mathrm{MnF}_{2}$. These transitions are $\alpha$-polarization active. A small CD signal is observed that is clearly related to this transition. The zero field splittings for the sidebands were determined to be $\Delta \omega\left(\sigma_{1}\right)=0.07 \mathrm{~cm}^{-1}$ and $\Delta \omega\left(\sigma_{2}\right)=-0.05 \mathrm{~cm}^{-1}$, respectively.

The negative sign in $\Delta \omega\left(\sigma_{2}\right)$ signifies that the sense of the splitting in this sideband is out of phase with that of $\sigma_{1}$. Such a change in the sense of splitting is also observed when uniaxial stress is applied to $\mathrm{MnF}_{2}$ along the [110] direction in the basal plane. The behavior of the $\mathrm{CD}$ was studied as a function of basal plane stress allowing the intrinsic strain in the basal plane of ordered $\mathrm{MnF}_{2}$ to be estimated: $\Sigma=\Delta d / d=1.2 \cdot 10^{-5}$. This result highlights the advantages of chiral techniques for exploring interactions in antiferromagnets.

$\mathrm{FeF}_{2}$ was studied in the $21500-28500 \mathrm{~cm}^{-1}$ spectral region. The observed transitions without exception exhibited MCD signals consistent with a simple sublattice splitting and with a linear dependence on magnetic field. No CD signals were encountered in any of the $\alpha$-active transitions investigated. The sensitivity of our apparatus places an upper limit of $10^{-3} \mathrm{~cm}^{-1}$ on any splitting that might occur in this compound at $4 \mathrm{~K}$.

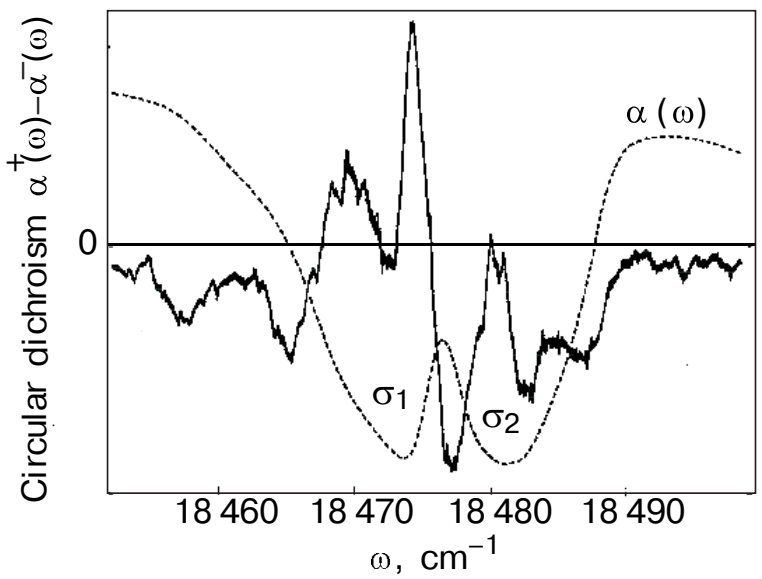

Fig. 2. The absorption and $\mathrm{CD}$ spectra of the so-called $\sigma_{1}$ and $\sigma_{2}$ sidebands of $\mathrm{MnF}_{2}$ at $2 \mathrm{~K}$; these traces connect the observed $\mathrm{CD}$ to the magnon sidebands. 


\section{Raman optical activity}

Raman optical activity is observed experimentally in the difference in a Raman line intensity when excited by right and left circularly polarized light, respectively. The circular intensity difference (CID) is normally used to quantify ROA [14]:

$$
\Delta_{\alpha}=\frac{I_{\alpha}^{R}-I_{\alpha}^{L}}{I_{\alpha}^{R}+I_{\alpha}^{L}}
$$

where $I_{\alpha}^{R}$ and $I_{\alpha}^{L}$ represent the scattered light intensity with $\alpha$ polarization due to incident right $(R)$ or left $(L)$ circularly polarized light. The normalization term permits a simple comparison of data from different spectroscopic systems. One major difficulty of ROA measurements is the sensitivity of the technique to intrinsic or extrinsic depolarization effects. Care must be taken to account for any birefringent effects in the steering and focusing optics. Additionally, the crystal itself can contribute to significant depolarization effects that appear as CID in spectra that normally would not be expected to produce any difference. We will discuss this effect in more detail in the next Section of the paper.

Fundamentally, the source of Raman scattering is the modulation of the induced polarizability of a medium by fundamental excitations in the medium. To couple the incident light to the medium, its susceptibility is expanded in terms of phonon or magnon operators. This results in a modulation of the polarizability at the frequency of the fundamental excitation. In the case of magnons, the inclusion of spin operators results in a change of the overall angular momentum component along the $z$ axis: $\Delta m_{j}= \pm 1$. Figure 3 illustrates the important mechanism for magnon light scattering from antiferromagnetic fluorides. The energy levels shown in the picture belong to the transition metal ions on the two sublattices. These transitions obey the same selection rules that contribute to $\mathrm{CD}$ or MCD signals. The circularly polarized light couples to different sublattices in the Raman process as well, thus providing a way of distinguishing between the

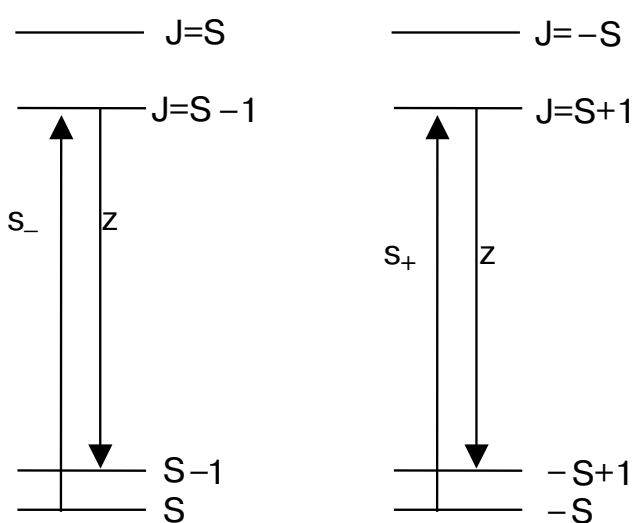

Fig. 3. The electronic levels of the magnetic ions in antiferromagnetically ordered rutile materials responsible for the observed ROA signal. The two sets of lines correspond to the two antiferromagnetic sublattices. In both cases a magnon is generated during the scattering process.

two sublattices. The figure shows energy levels for both sublattices in the medium and illustrates that left and right circular polarizations create magnons on different sublattices.

Symmetry arguments can be applied to determine selection rules for the various scattering processes and are summarized in the form of Raman scattering tensors. The scattering tensors for the tetragonal antiferromagnetic fluorides are presented in Table 1.

The tensors have been transformed so that they are appropriate for $[R, L, z]$ incident polarizations and $[x, y, z]$ scattered polarizations. The Raman tensors describe the scattered electric field amplitudes and thus the scattered light intensity in a specific polarization is calculated through multiplication of the tensor by its conjugate value. To describe the expected CID spectrum, we need to calculate the difference between two intensity terms. For example, the $A_{1 g}$ phonon mode should not generate a CID signal for scattered light polarized along the $x$ axis:

$$
\Delta_{x}\left(A_{1 g}\right)=\frac{I_{x}^{R}-I_{x}^{L}}{I_{x}^{R}+I_{x}^{L}}=\frac{a^{2}-a^{2}}{a^{2}+a^{2}}=0 .
$$

Table 1. The Raman tensors for circularly polarized incident light that is analyzed for linearly polarized light scattered at 90 degrees.

$$
\begin{aligned}
& A_{1 g}=\frac{1}{\sqrt{2}}\left[\begin{array}{ccc}
a & a & 0 \\
-i a & i a & 0 \\
0 & 0 & \sqrt{2} b
\end{array}\right] \\
& B_{1 g}=\frac{1}{\sqrt{2}}\left[\begin{array}{ccc}
c & c & 0 \\
-i c & i c & 0 \\
0 & 0 & 0
\end{array}\right] \\
& B_{2 g}=\frac{1}{\sqrt{2}}\left[\begin{array}{ccc}
-i e & i e & 0 \\
e & e & 0 \\
0 & 0 & 0
\end{array}\right] \\
& \Gamma_{3}^{+}+\Gamma_{4}^{+}\left[\frac{|+1\rangle}{|-1\rangle}\right]=\frac{1}{\sqrt{2}}\left[\begin{array}{ccc}
0 & 0 & \pm \sqrt{2} \delta \\
0 & 0 & i \sqrt{2} \delta^{*} \\
\left( \pm \varepsilon+\varepsilon^{*}\right) & \left( \pm \varepsilon-\varepsilon^{*}\right) & 0
\end{array}\right] \\
& E_{g}(1)=\frac{1}{\sqrt{2}}\left[\begin{array}{ccc}
0 & 0 & 0 \\
0 & 0 & \sqrt{2} d \\
-i d & i d & 0
\end{array}\right] \\
& E_{g}(2)=\frac{1}{\sqrt{2}}\left[\begin{array}{ccc}
0 & 0 & \sqrt{2} d \\
0 & 0 & 0 \\
d & d & 0
\end{array}\right]
\end{aligned}
$$


In the case of the magnon scattering we can calculate the CID for each sublattice separately. In the case of the $\Gamma_{3}^{+}$band, we obtain the following CID expression:

$$
\Delta_{z}\left(\Gamma_{3}\right)=\frac{\left(\varepsilon^{*} \varepsilon+\varepsilon^{*}+\varepsilon^{*} \varepsilon+\varepsilon^{2}\right)-\left(\varepsilon^{*} \varepsilon-\varepsilon^{* 2}+\varepsilon^{*} \varepsilon-\varepsilon^{2}\right)}{\left(\varepsilon^{*} \varepsilon+\varepsilon^{* 2}+\varepsilon^{*} \varepsilon+\varepsilon^{2}\right)+\left(\varepsilon^{*} \varepsilon-\varepsilon^{* 2}+\varepsilon^{*} \varepsilon-\varepsilon^{2}\right)}=\frac{\varepsilon^{* 2}+\varepsilon^{2}}{2\left(\varepsilon^{*} \varepsilon\right)} .
$$

Likewise for the $\Gamma_{4}^{+}$magnon band we get a similar result:

$$
\Delta_{z}\left(\Gamma_{4}\right)=-\frac{\varepsilon^{* 2}+\varepsilon^{2}}{2\left(\varepsilon^{*} \varepsilon\right)}
$$

The negative sign reflects the coupling of left circular polarization to this particular sublattice. Notice that if the energies of the two magnon bands are equal and the line profiles equivalent, the total CID is the sum of these terms and will be zero. The application of a magnetic field lifts the degeneracy of the magnon branches. As a result, the CID of the two branches will no longer completely cancel. This result is analogous to the differences observed between MCD and $\mathrm{CD}$ spectra. The important distinction is that the Raman spectra are determined only by the energies of the magnon bands with no interference from the excited states of the ion.

\section{Raman optical activity experiments}

The experimental arrangement, shown in Fig. 4, used the standard $90^{\circ}$ Raman scattering geometry. The incident beam was the 488-nm line of a continuous-wave Spectra Physics 166 argon-ion laser. The focused laser power density at the sample was $150 \mathrm{~kW} / \mathrm{cm}^{2}$, well below the damage threshold of the materials used. A Conoptics 370 electro-optic modulator (EOM) was used to alternate between right and left circular polarization. The scattered light was dis-

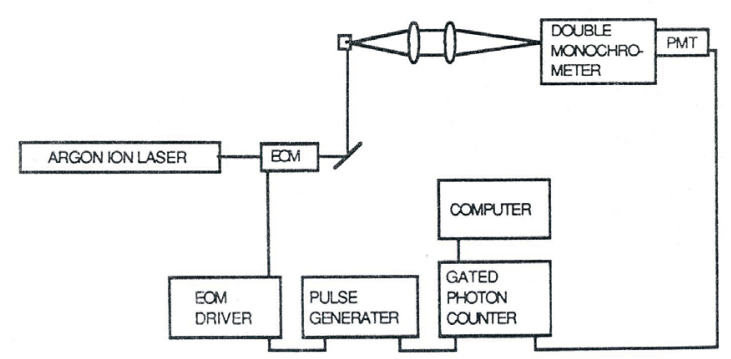

Fig. 4. The experimental setup for the ROA measurements. The laser light was directed vertically through the sample to align the beam path with the entrance slit of the spectrometer. persed by a Spex 1401, 0.85-m double monochromator and detected using a cooled RCA 31034-A02 photomultiplier. The signal was measured using a Stanford SR-400 dual-gated photon counter and stored on a computer. The signals counted on the two gates, corresponding to the two incident polarizations, were stored separately, permitting both $I^{R}-I^{L}$ and $I^{R+}+I^{L}$ to be easily calculated. Low temperatures were achieved using a Cryosystems closed-cycle refrigerator. A permanent magnet assembly located inside the refrigerator applied a constant magnetic field parallel to the crystal $c$ axis. The magnets were drilled through so that the incident laser beam could be focused in the crystal co-linear with the applied field and allowed to pass on through the refrigerator.

The crystals used in this study were the rutile structure fluorides $\mathrm{FeF}_{2}$ and $\mathrm{MgF}_{2}$. These materials are uniaxial, with $\mathrm{FeF}_{2}$ undergoing an antiferromagnetic phase transition at $T_{N}=78 \mathrm{~K}$. The $\mathrm{MgF}_{2}$ crystal orientation was determined from $\mathrm{x}$-ray Laue-diffraction measurements before the cuboid sample was cut (to within a $1^{\circ}$ accuracy) and polished. The $c$-axis orientation for the $\mathrm{FeF}_{2}$ sample was found using a polariscope. In addition, a polished crown glass cube was used as a reference material to check for instrumental artifacts.

\section{Rayleigh and Raman CID in $\mathrm{MgF}_{2}$ and $\mathrm{FeF}_{2}$}

Our measurements of the magnon and phonon CID in $\mathrm{FeF}_{2}$ generated several signals that were difficult to understand initially. Figure 5 shows the difference spectrum $I^{R}-I^{L}$ as well as the separate $I^{R}$ and $I^{L}$ spectra from $\mathrm{FeF}_{2}$ with no applied magnetic field. What is curious about these measurements is that the magnon exhibits no apparent differential scattering but the $B_{1 g}$ phonon mode does. When the spectrum is enlarged to encompass all of the phonon modes and the Rayleigh line, we observe a similar CID spectrum for the $A_{1 g}$ and $B_{2 g}$ modes and the Rayleigh line but the sign of the difference signal is opposite to that of the $B_{1 g}$ mode. Referring back to the Raman tensors, no CID is expected from any of the phonon modes nor should Rayleigh scattering exhibit CID. $\mathrm{MgF}_{2}$ is also a rutile structure fluoride, but it does not exhibit any magnetic ordering. It showed a similar differential scat- 


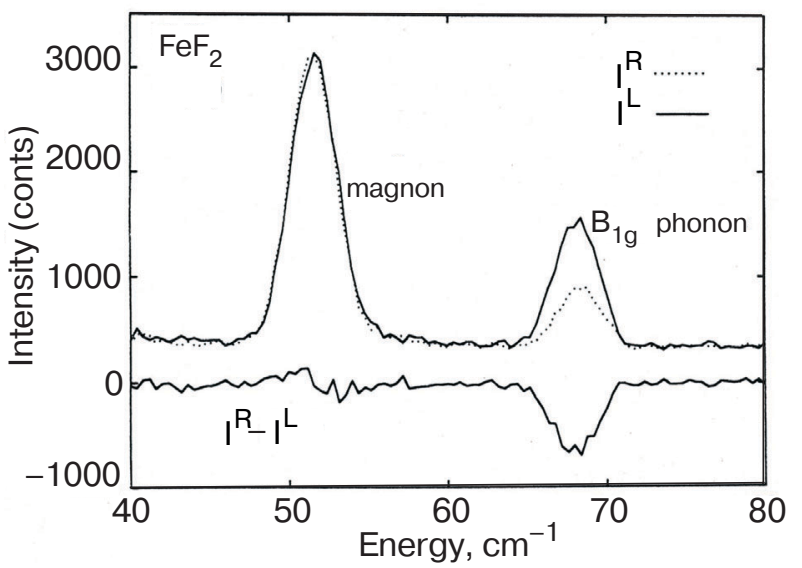

Fig. 5. The ROA spectrum of $\mathrm{FeF}_{2}$ at $4 \mathrm{~K}$. The presence of a CID spectrum in the $B_{1 g}$ phonon mode indicates a misalignment of the incident laser and the crystalline $\mathrm{c}$ axis.

tering pattern. An isotropic glass sample was used to check for instrumental artifacts and none were found.

To explain the CID spectra of the phonons we looked carefully at the effects of birefringence in the crystal on the scattered intensity. Light propagating along the $c$-axis will experience an isotropic index of refraction so that no change in the polarization occurs as it moves through the sample. A slight misalignment results in distinct indices of refraction parallel and perpendicular to the plane containing the $c$ axis. As a result, circularly polarized light becomes elliptically polarized as it moves through the sample. The changes in polarization affect the relative amplitude of the $x$ and $y$ components of the incident light. The crucial point is that changes of $I_{x}$ and $I_{y}$ are different for left and right circular polarization so that any scattering that depends on only one of these terms will exhibit a CID spectrum. This result permits a very sensitive tool for aligning the samples for ROA measurements so that intrinsic birefringence effects can be eliminated.

\section{Raman optical activity of Magnons in $\mathrm{FeF}_{2}$}

Figure 6 presents the conventional Raman and ROA spectrum of the one-magnon line of $\mathrm{FeF}_{2}$ in an applied magnetic field. The modest applied field, $B<0.6 \mathrm{~T}$, is too small to observe the splitting of the magnon branches within the magnon line width using conventional (linearly polarized light) Raman techniques. However, the magnon line exhibits a clear CID spectrum. To determine the energy difference between the two branches we fit the data using offset Gaussian line shapes to describe the one magnon scattering peak. In this manner we can calculate the energy difference as a function of temperature. Figure 7 shows the temperature dependence of the frequency splitting between the two magnon branches in $\mathrm{FeF}_{2}$. As the temperature

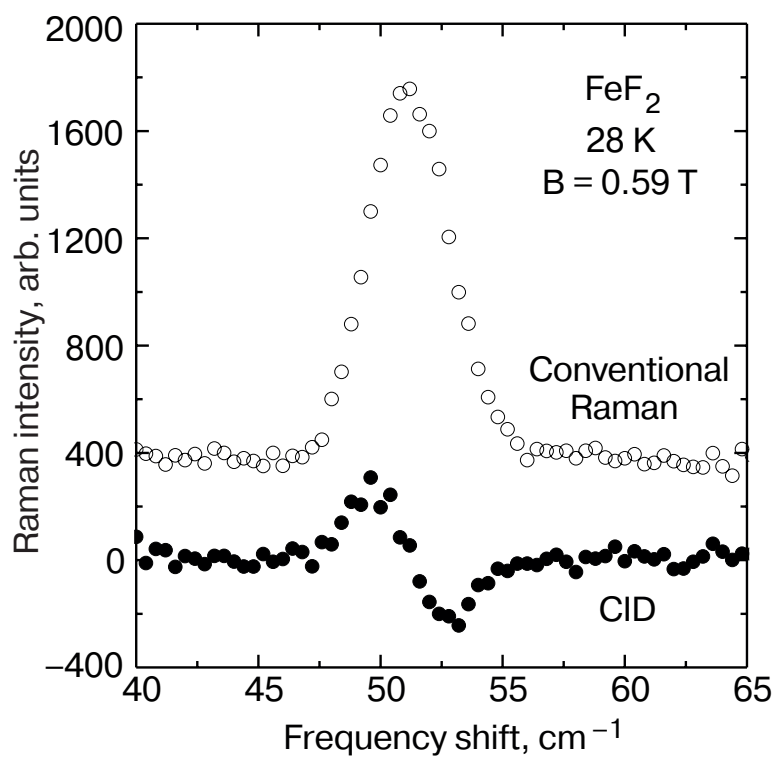

Fig. 6. The low temperature $\mathrm{ROA}$ spectrum of $\mathrm{FeF}_{2}$ in the presence of an applied magnetic field. The upper trace shows the conventional Raman spectrum. The lower trace is the measured CID spectrum.

increases, the energy splitting between the branches decreases, while the linewidth increases resulting in a larger uncertainty for the energy splitting between the branches.

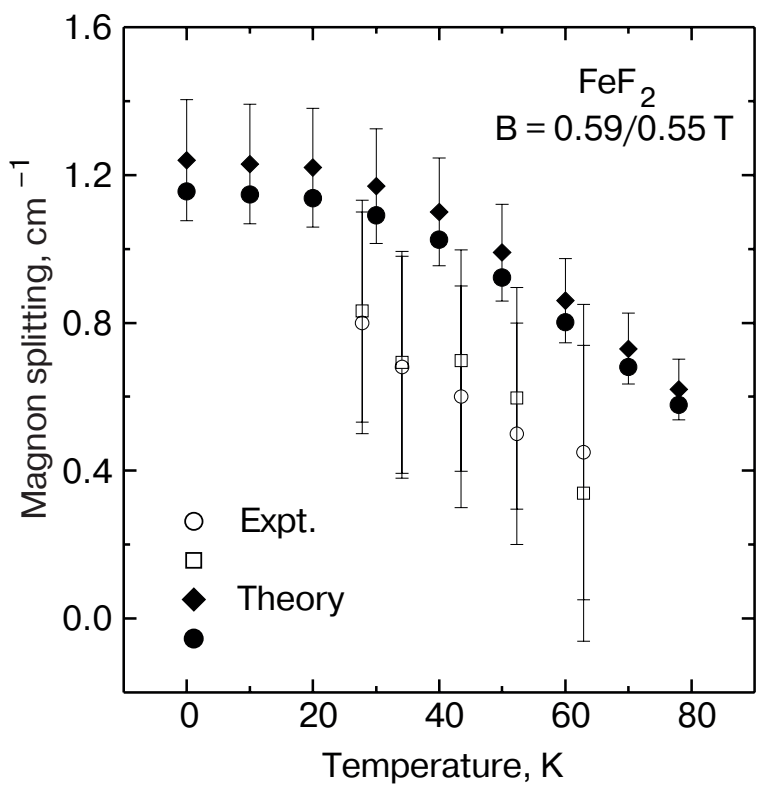

Fig. 7. The temperature dependence of the magnon branch splitting obtained from ROA measurements in $\mathrm{FeF}_{2}$. The theoretical points are calculated using applied fields of 0.59 (filled diamonds) and $0.55 \mathrm{~T}$ (filled circles). The error bars on the theoretical points derive largely from the uncertainty in the applied magnetic field strength. 
Theoretically, [28] the energy splitting between the two branches is given by

$$
\Delta \omega(T)=2\left[g \mu_{B}-J \chi_{\|}\right] H
$$

where $H$ is the applied magnetic field along the crystal $c$-axis, $\chi_{\|}$is the parallel susceptibility per spin site, $\mu_{B}$ is the Bohr magneton, $J$ is the dominant nearest neighbor exchange, and $g$ is the Lande $g$ factor. At low temperatures, $\chi_{\|}$is nearly zero so:

$$
\Delta \omega(T=0)=2 g \mu_{B} H
$$

whereas at temperatures near $T_{N}, \chi_{\|}=g \mu_{B} / 2 J$ so:

$$
\Delta \omega\left(T=T_{N}\right)=g \mu_{B} H .
$$

This theory predicts that the splitting between the branches should change by a factor of two when going from low temperature to the phase transition temperature. Using parameters obtained from the experiment or the literature the theoretical curve shown in Fig. 7 was obtained. The theoretical curve accurately describes the temperature variation of the energy splitting between the magnon bands, but overall there is a general offset between the measured data and the theoretical curve. The primary uncertainty for this measurement arises from determining the actual value of the magnetic field inside the refrigerator. The magnetic field of $0.59 \mathrm{~T}$ for the permanent magnet gap was measured at room temperature. The magnet will be at a much lower temperature during the Raman measurement, but the affects of the lower temperature on the gap field strength is not known.

These measurements highlight the sensitivity of this technique in measuring magnon branch splittings. The line widths of the two peaks at $62 \mathrm{~K}$ were greater than $10 \mathrm{~cm}^{-1}$, but the separation between the peaks was only $0.4 \mathrm{~cm}^{-1}$.

For a simple antiferromagnetic, we would not expect to observe any CID spectrum in zero applied field ROA measurements. However, careful measurements revealed a small but repeatable CID spectrum in $\mathrm{FeF}_{2}$. Figure 8 shows the CID spectrum and the separate $I^{R}$ and $I^{L}$ Raman signals of $\mathrm{FeF}_{2}$. Care was taken to eliminate all of the depolarization effects by using the Rayleigh scattering spectrum to ensure alignment of the crystal $c$-axis with the incident laser light. The solid line fit determined the zero field splitting between the two magnon branches to be $\Delta \omega=(0.09 \pm 0.02) \mathrm{cm}^{-1}$. To our knowledge this result is the first measurement of a zero field splitting of the magnon branches in antiferromagnets using Raman scattering. Again the high sensitivity and resolution of this technique is highlighted in obtaining this result.

Finally, ROA measurements of the two-magnon scattering peak in $\mathrm{FeF}_{2}$ showed a null result. The lack

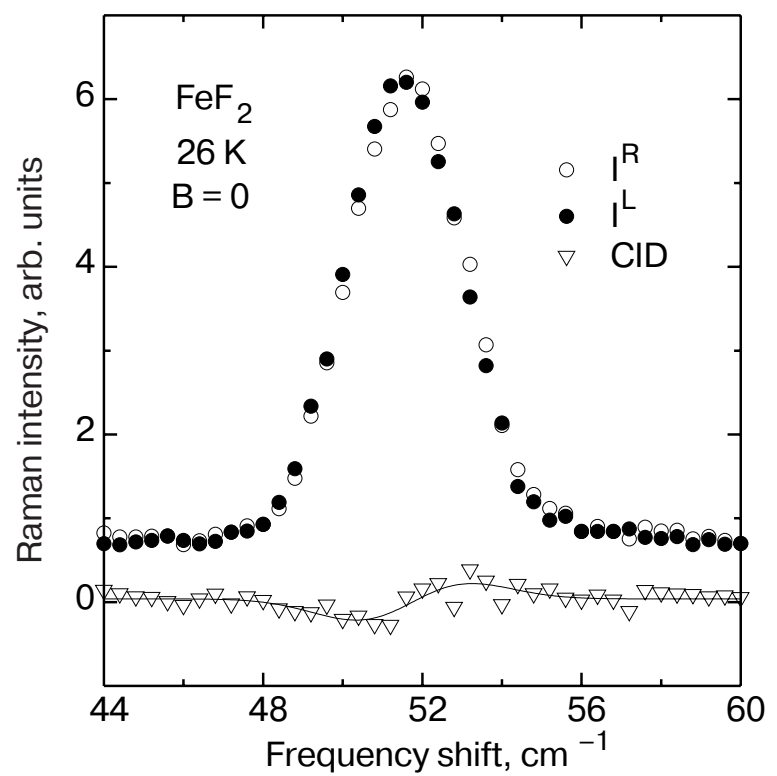

Fig. 8. The zero-field ROA spectrum of the one magnon line in $\mathrm{FeF}_{2}$. The solid line is a fit modeling the small CID apparent in this spectrum.

of any CID signal confirms that the dominant mechanism for two-magnon scattering involves exchange coupled magnons on opposite sublattices. Other possible sources of two-magnon scattering are higher order processes that would be expected to be much weaker than the one-magnon scattering. Multiple spin-flip transitions have been observed in dilute magnetic semiconductors in the paramagnetic phase under resonance excitation conditions. These transitions were observed in a forward scattering geometry where the scattered light was analyzed for the circular polarization as well. The intensities of the two spin flip signals are much lower than the single spin flip signals consistent with a higher order interaction.

\section{Magnetic dipole-dipole interactions}

In 1963 Loudon and Pincus [25] examined the effect of the classical dipole-dipole interaction between magnetic moments on the spin wave spectrum of a simple uniaxial antiferromagnet. In the absence of an applied magnetic field, as discussed above, the spin wave branches are degenerate in these systems. The magnon dispersion relation is given by

$$
\omega \equiv \gamma\left[H_{A}^{2}+2 H_{A} H_{E}+2 H_{E}^{2} b^{2} k^{2}\right]^{1 / 2}
$$

where $\gamma=g \mu_{B}$ is the gyromagnetic ratio, $H_{A}$ is the uniaxial single-ion internal anisotropy field and $H_{E}$ is the effective exchange field; $k$ is the wave vector and $b=\mathrm{a} z^{-1 / 2}$ with $a$ the nearest neighbor distance and $z$ the number of nearest neighbors. On inclusion of the 
dipolar fields, Eq. (8) splits into two modes with their dispersions given by:

$$
\omega_{1}=\gamma\left[H_{A}^{2}+2 H_{E} H_{A}+2 H_{E}^{2} b^{2} k^{2}\right]^{1 / 2},
$$

$\omega_{2}=\gamma\left[H_{A}^{2}+2\left(H_{A}+H_{E} b^{2} k^{2}\right)\left(H_{E}+4 \pi M_{S} \sin ^{2} \theta_{k}\right)\right]^{1 / 2}$

where $M_{s}$ is the sublattice magnetization and $\theta_{k}$ is the angle between the easy axis $(z)$ of magnetization and the direction of propagation of the spin wave, $\mathbf{k}$. Only the frequency of the second mode is affected by the dipolar interaction. For spin waves with $\mathbf{k}=0$, Eqs. (9) and (10) reduce to

$$
\begin{gathered}
\omega_{1}=\gamma\left[H_{A}\left(H_{A}+2 H_{E}\right)\right]^{1 / 2} \\
\omega_{2}=\gamma\left[H_{A}\left(H_{A}+2 H_{E}+8 \pi M_{S} \sin ^{2} \theta_{0}\right)\right]^{1 / 2} .
\end{gathered}
$$

In the case of longitudinal spin waves, $\theta_{0}=0$, and there is no dipolar effect. For the transverse spin waves, however, $\theta_{0}=\pi / 2$, and the dipolar effects are at a maximum.

Subsequently, Harris [26] has shown that Eq. (10) is an approximate result and has derived the full expression for the spin wave dispersion in the presence of a dipolar field. However, the difference between Eq. (10) and the complete result only becomes important when $4 \pi M_{S}$ is comparable to $H_{E}$, which is not the case here.

The Loudon and Pincus calculation is based on a molecular field approach that neglects the affect of the Lorentz field of one sublattice upon the other sublattice. When this effect is included, White [27] showed that the resonant frequencies for $\mathbf{k}=0$ are given by:

$$
\begin{gathered}
\omega(k=0)_{1,2}= \\
= \pm \gamma\left[H_{A}\left(H_{A}-\frac{8}{3} \pi M_{S}\right)+2 H_{A}\left(H_{E}+4 \pi M_{S} N_{x}\right)\right]^{1 / 2},
\end{gathered}
$$

$$
\begin{gathered}
\omega(k=0)_{3,4}= \\
= \pm \gamma\left[H_{A}\left(H_{A}-\frac{8}{3} \pi M_{S}\right)+2 H_{A}\left(H_{E}+4 \pi M_{S} N_{y}\right)\right]^{1 / 2}
\end{gathered}
$$

and for $\mathbf{k} \neq 0$ by

$$
\begin{gathered}
\omega(k)_{1,2}= \pm \gamma\left[H_{A}^{2}+2 H_{A} H_{E}+H_{E}^{2}\left(1-\gamma_{k}^{2}\right)+\right. \\
\left.+\frac{8}{3} \pi M_{S}\left[H_{A}+H_{E}\left(1-\gamma_{k}\right)\right]\right]^{1 / 2}, \\
\omega(k)_{3,4}= \pm \gamma\left[H_{A}^{2}+2 H_{A} H_{E}+H_{E}^{2}\left(1-\gamma_{k}^{2}\right)+\right. \\
\left.+8 \pi M_{S}\left(\frac{1}{3}-\sin ^{2} \theta_{k}\right)\left[H_{A}+H_{E}\left(1-\gamma_{k}\right)\right]\right]^{1 / 2}
\end{gathered}
$$

where $\mathbf{N}$ is the demagnetization dyadic [27], and $\gamma_{k}$ is defined as $(1 / z) \Sigma_{\delta} \exp (\mathrm{ik} \cdot \delta)$, where the $\delta$ 's are the vectors to the $z$ nearest neighbors.

Notice that for the uniform spin precession $(\mathbf{k}=0)$ in a sphere $\left(N_{x}=N_{y}=N_{z}=1 / 3\right)$, the Lorentz field just cancels the surface demagnetizing field. Therefore, there is no shift in the resonant frequency as compared to non-dipolar cases. The symmetric occurrence of $N_{x}$ and $N_{y}$ in Eq. (12) is due to the fact that the normal modes labeled 1,2 and 3, 4 are characterized by net transverse magnetizations that are linearly polarized in the $x$ and $y$ directions, respectively. Notice also that although the 1, 2 spin wave modes are independent of $\theta$ they are shifted by the Lorentz field.

Since the samples used in the optical studies had rectangular cross sections in the basal plane, there will be dipolar splittings of the magnons. Thus, from Eq. (12),

$$
\Delta \omega_{k=0}=\omega_{1}-\omega_{3}=\frac{4 \pi M_{s} H_{A}\left(N_{x}-N_{y}\right)}{\sqrt{2 H_{A} H_{E}+H_{A}^{2}}} .
$$

The demagnetizing factors are given by integrals over the surface [27]. In order to obtain estimates of these splittings we approximate $N_{x}$ and $N_{y}$ by $1 / 2$ and $1 / 4$, respectively, based on the sample dimensions. The parameter values used in the calculations and the resulting splittings are listed for $\mathrm{MnF}_{2}, \mathrm{CoF}_{2}$, and $\mathrm{FeF}_{2}$ in Table 2.

\section{Comparison of theory with experiment}

\section{Optical circular dichroism}

The calculated splittings given in Table 2 are quite small compared with the magnon energies in these compounds. Given the nature of the natural line widths, splittings of this magnitude are difficult to measure and they have been resolved only recently using Raman spectroscopy $[11,15]$.

The calculated values for $\Delta \omega$ in $\mathrm{MnF}_{2}$ and $\mathrm{CoF}_{2}$ (see Table 2) are of similar magnitude to those found experimentally for the magnon sidebands in the visi- 
ble region: $0.02 \mathrm{~cm}^{-1}$ for $\mathrm{CoF}_{2}$ and 0.07 and $0.05 \mathrm{~cm}^{-1}$ for $\mathrm{MnF}_{2}$. Thus the origin of the observed splittings reported in the $\mathrm{CD}$ measurements is well encompassed by the effects of the magnetic dipole-dipole interaction in these simple rutile antiferromagnets. The size of the splittings detected by spontaneous magnetic $\mathrm{CD}$ cannot be compared directly with those calculated in Table 2, as the processes involved in the formation of magnon sidebands are not as simple and direct as those encountered in Raman scattering. For $\mathrm{MnF}_{2}$, for example, the sideband involves a combination of an exciton on one sublattice with essentially zero dispersion [25] with a magnon on the other with nearly 50 $\mathrm{cm}^{-1}$ dispersion; $\mathbf{k}$ conservation requires the creation of exciton-magnon pairs with equal and opposite wave vectors from throughout the Brillouin zone. Although the splitting is mostly uniform across the Brillouin zone, the CD is, in effect, sampling the derivative of the joint density of states. It follows that an accurate calculation of the $\mathrm{CD}$ requires the use of Eq. (13), but the theory needs to be extended to include specific symmetry points in the Brillouin zone and an appropriate summation over the entire zone.

Conceivably, another possible cause of CD in these compounds could be a slight tilting of the spins away from their ordered positions along the c-axis. However, at low temperatures and in zero applied magnetic field, all experimental results to date point to collinear spin alignment in all of these compounds [29].

\section{Raman circular intensity difference}

In the Raman equivalent of the $\mathrm{CD}$ measurements discussed above, a $\Delta \omega$ of $(0.09 \pm 0.02) \mathrm{cm}^{-1}$ was observed in $\mathrm{FeF}_{2}$ at low temperatures using a $90^{\circ}$ scattering geometry. From Eq. (11), the theoretical splitting for magnons propagating at approximately $45^{\circ}$ to the $c$ (z) axis is $\Delta \omega=0.15 \mathrm{~cm}^{-1}$, which considering the expected large uncertainty in $M_{\mathrm{S}}$ is in good agreement with the experimental results. Including the Lorenz field correction, Eq. (14) gives $\Delta \omega=0.065 \mathrm{~cm}^{-1}$ (see Table 2), which is in even closer agreement with experiment. The zero-field splitting of the magnon branches observed in $\mathrm{FeF}_{2}$ is thus attributed to the magnetic dipole-dipole interactions that can be important for long wavelength magnons [27].

Another way to quantitatively describe the interaction is to define it in terms of an effective anisotropy field, $H^{\prime}$, that is treated in the same manner as an external magnetic field. In this approach the energy of the magnons shows up in much the same form as presented in Eq. (6) except the splitting is now due to $H^{\prime}$.

$$
\Delta \omega(T=0)=2 \gamma H^{\prime}=0.09 \mathrm{~cm}^{-1}
$$

Table 2. Magnetic parameters at low temperature for various transition metal fluorides possessing the rutile structure. The calculated dipole-dipole induced splittings, $\Delta \omega_{\mathrm{k}=0}$ and $\Delta \omega_{k=\pi / 2}$, are also given.

\begin{tabular}{c|c|c|c}
\hline \hline Parameter & $\begin{array}{c}\mathrm{MnF}_{2} \\
{[28-30]}\end{array}$ & $\begin{array}{c}\mathrm{FeF}_{2} \\
{[29,31]}\end{array}$ & $\begin{array}{c}\mathrm{CoF}_{2} \\
{[29,32]}\end{array}$ \\
\hline \hline Effective spin $S$ & $5 / 2$ & 2 & $1 / 2$ \\
$H_{E}(T)$ & 51.5 & 53.313 & 37.6 \\
$H_{A}(T)$ & 0.84 & 19.745 & 9.54 \\
$g$ & 2.00 & 2.25 & 2.80 \\
$\gamma\left(\mathrm{cm}^{-1} / \mathrm{T}\right)$ & 0.934 & 1.05 & 1.31 \\
$M_{S}(\mathrm{~T})$ & 0.060 & 0.056 & $\sim 0.06$ \\
$\Delta \omega_{k=0}\left(\mathrm{~cm}^{-1}\right)$ & 0.016 & 0.065 & 0.083 \\
$\Delta \omega_{k=\pi / 2}\left(\mathrm{~cm}^{-1}\right)$ & 0.02 & 0.08 & 0.12 \\
\hline \hline
\end{tabular}

Using the parameter value $\gamma=1.05 \mathrm{~cm}^{-1} / T$ (Table 2) we calculate the anisotropy field to be: $H^{\prime}=(0.04 \pm$ $0.01) \mathrm{T}$.

By comparing Figs. 8 and 6 we see that the CID spectra have opposite signs for the case of $H=H^{\prime}$ and $H=H_{\text {applied }}+H^{\prime}$, respectively. Since the energy splitting of the two magnetic sublattices switches sign with the external field, the direction of the applied field must be opposite to the direction of the effective anisotropy field. Going back to the temperature dependence of the magnon branch splitting we can now correct for the presence of the effective anisotropy field in the material. Equation (5) becomes

$$
\begin{aligned}
\Delta \omega & (T)=2\left[g \mu_{B}-J \chi_{\|}\right]\left(H_{\text {applied }}-H^{\prime}\right)= \\
& =2\left[g \mu_{B}-J \chi_{\|}\right](0.55 \mathrm{~T}) .
\end{aligned}
$$

The second theoretical curve shown on Fig. 7 uses this new value for the magnetic field and it gives better agreement with the measured data.

\section{Conclusions}

The magnon sideband CD observed in rutile structure antiferromagnets can be quite readily explained as being the result of magnetic dipole-dipole interactions breaking the degeneracy of the two spin wave branches. These optical measurements performed three decades ago are seen now to represent the first observation of such splittings, as first predicted by Loudon and Pincus in 1963. It would be informative to perform further experiments to confirm the $\sin ^{2} \theta$ dependence of the splitting by varying the angle $\theta_{0}$ from 0 to $\pi / 2$, i.e., the incident light direction is varied from along to perpendicular to the easy axis, and also the sample shape effect on the splitting. 
The ability to measure an effective anisotropy field in $\mathrm{FeF}_{2}$ highlights the sensitivity of ROA measurements for determining small splittings between the magnon branches in antiferromagnets. The measurement of such a splitting due to magnetic dipole-dipole interactions in $\mathrm{FeF}_{2}$ has permitted a final resolution of questions concerning the origins of $\mathrm{CD}$ in optical measurements completed over thirty years ago. The high sensitivity of ROA also permits the study of antiferromagnetic branch splittings for low applied magnetic fields.

The precise experimental work of Professor F.L. Scarpace and Dr. Y.H. (Russ) Wong from thirty years ago is noted with appreciation. We thank Professors R. M. White and M. G. Cottam for useful discussions and the National Science Foundation for continued support spanning these three decades.

1. R.L. Greene, D.D. Sell, W.M. Yen, A.L. Schawlow, and R.M. White, Phys. Rev. Lett. 15, 656 (1965).

2. J.W. Halley and I. Silvera, Phys. Rev. Lett. 15, 654 (1965).

3. Y. Tanabe, T. Moriya, and, S. Sugano, Phys. Rev. Lett. 15, 1023 (1965).

4. S.J. Allen, R. Loudon, and P.L. Richards, Phys Rev. Lett. 16, 643 (1966).

5. P.A. Fleury, S.P.S. Porto, L.E. Cheeseman, and H.J. Guggenheim, Phys. Rev. Lett. 17, 84 (1966).

6. P.A. Fleury and R. Loudon, Phys. Rev. 166, 514 (1968).

7. M.G. Cottam and D.J. Lockwood, Light Scattering in Magnetic Solids, Wiley, New York (1986).

8. F.L Scarpace, M.Y. Chen, and W.M Yen, J. Appl. Phys. 42, 1655 (1971).

9. M.Y. Chen, F.L. Scarpace, M.W. Passow, and W.M. Yen, Phys. Rev. B4, 1332 (1971).

10. Y.H. Wong, C.D. Pfeifer, and W.M Yen, in: Magnetism and Magnetic Materials (1972) C.D. Graham, Jr. and J.J. Rhyne (eds.) AIP Conference Proc. No. 10 AIP, New York (1973) p. 1960.

11. D.J. Lockwood, R.M White, and W.M. Yen, Phys. Rev. B69, 174413 (2004).

12. W.M. Yen and Y. Oka, Bussei Kenkyu 13, 507 (1972).

13. L.D. Barron, M.P. Bogaard, and A.D. Buckingham, J. Am. Chem. Soc. 95, 603 (1973).
14. L.D. Barron and A.D. Buckingham, Mol. Phys. 20, 1111 (1971).

15. L.D. Barron, Molecular Light Scattering and Optical Activity, Cambridge University Press, Cambridge (1982).

16. L.D. Barron, L. Hecht, In: J. Nakanishi, N. Berova, and R.W. Woody (eds.) Circular Dichroism Principles and Applications, VCH Publishers, New York (1994).

17. Petrou, D.L. Peterson, S. Venugopalan, R.R. Galazka, A.K. Ramdas, and S. Rodriguez, Phys. Rev. B27, 3471 (1983)

18. D.L. Peterson, D.U. Barthololmew, A.K. Ramdas, and S. Rodriguez, Phys. Rev. B31, 7932 (1985).

19. K.R. Hoffman, W. Jia, andW.M. Yen, Opt. Lett. 15 332 (1990).

20. K.R. Hoffman, W. Jia, and W.M. Yen, in: J.R. Durig and J.F. Sullivan (eds.), Proceedings of the $12^{\text {th }}$ International Conference on Raman Spectroscopy, Wiley, New York (1990), p. 864.

21. K.R. Hoffman, W.M. Yen, and D.J. Lockwood in: N.T. Yu and X.Y. Li (eds.), Proceedings of the $14^{\text {th }}$ International Conference on Raman Spectroscopy, Wiley, Chichester (1994), p. 1078.

22. K.R. Hoffman, W.M. Yen, and D.J. Lockwood in: W. Kiefer et. al. (eds.), Proceedings of the 13th International Conference on Raman Spectroscopy, Wiley, Chichester (1992), p. 766

23. K.R. Hoffman, W.M. Yen, D.J. Lockwood, and P.E. Sulewski, Phys. Rev. B49, 182 (1994).

24. D.J. Lockwood, K.R. Hoffman, and W.M. Yen, J. Lumin. 100, 147 (2002).

25. R. Loudon and P. Pincus, Phys. Rev. 132, 673 (1963).

26. A.B. Harris, Phys. Rev. 143, 353 (1966).

27. M.G. Cottam and D.J. Lockwood, Light Scattering in Magnetic Solids, Wiley, New York, (1986) Chapter 5.

28. D.D. Sell, R.L. Greene, and R.M. White, Phys. Rev. 158, 149 (1967).

29. R.A. Cowley and W.J.L. Buyers, Rev. Mod. Phys. 44, 406 (1972).

30. J.P. Kotthaus and V. Jaccarino, Phys. Rev. Lett. 28, 1649 (1972).

31. T. Dumelow, R.E. Camley, K. Abraha, and D.R. Tilley, Phys. Rev. B58, 897 (1998).

32.P.L. Richards, J. Appl. Phys. 35, 850 (1964). 